\title{
Avaliação da resiliência em adultos e idosos: revisão de instrumentos
}

\section{Assessment of resilience in adults and the elderly: A review of instruments}

\author{
Léia Gonçalves GURGEL ${ }^{1}$ \\ Rodrigo Della Méa PLENTZ \\ Maria Cristina Rodrigues Azevedo JOLY3 \\ Caroline Tozzi REPPOLD ${ }^{4}$
}

\begin{abstract}
Resumo
Este estudo revisa sistematicamente na literatura os instrumentos utilizados para a avaliação da resiliência em adultos e idosos. A pesquisa foi realizada em: MedLine, PsycINFO, Scopus, SciELO, Interscience databases, envolvendo os termos resilience, psychological, scales e validation studies. Foram incluídos estudos de avaliação da resiliência em indivíduos acima de 18 anos. A busca resultou em 59 estudos, sendo que apenas 16 preencheram os critérios de elegibilidade. Foram considerados 13 instrumentos validados para avaliação de resiliência. O total de 8689 indivíduos foi incluído nos 16 estudos, e a idade variou de 12 a 97 anos. Os testes apresentam principalmente estudos de precisão e são compostos por poucos itens. Poucos estudos de validade preditiva foram encontrados. Algumas pesquisas incluídas são específicas para populações indígenas, mulheres, veteranos de guerra e esquizofrênicos. São poucos os instrumentos disponíveis para avaliação da resiliência em adultos e idosos, sobretudo para uso em estudos nacionais.
\end{abstract}

Unitermos: Estudos de validação; Resiliência psicológica; Testes psicológicos.

\begin{abstract}
This study is a systematic review of the literature for the main instruments used in the assessment of resilience in adults and the elderly. A search was performed in: the MedLine (accessed via PubMed), PsycINFO, Scopus, SciELO, and InterScience databases. The search used the following terms: Resilience, Psychological, Scales, and Validation Studies. All the validation studies of instruments involving the evaluation of psychological resilience in individuals over 18 years were included. The search yielded 59 studies, however, only 16 studies met the eligibility criteria for the analysis. Thirteen instruments were considered valid for the assessment of resilience. A total of 8,689
\end{abstract}

\section{$\nabla \nabla \nabla$}

1 Universidade Federal de Ciências da Saúde de Porto Alegre, Programa de Pós-Graduação em Ciências da Saúde. R. Sarmento Leite, 245, Centro, 90050-170, Porto Alegre, RS, Brasil. Correspondência para/Correspondence to: L.G. GURGEL. E-mail: <leiagg@yahoo.com.br>.

2 Universidade Federal de Ciências da Saúde de Porto Alegre, Departamento de Fisioterapia, Programa de Pós-Graduação em Ciências da Reabilitação. Porto Alegre, RS, Brasil.

3 Universidade de Brasília, Instituto de Psicologia, Programa de Pós-Graduação em Processos de Desenvolvimento Humano e Saúde. Brasília, DF, Brasil.

4 Universidade Federal de Ciências da Saúde de Porto Alegre, Departamento de Psicologia, Pós-Graduação em Ciências da Reabilitação. Porto Alegre, RS, Brasil. 
subjects, between 12 to 97 years of age, were included in the 16 studies. The instruments contained few items. Few studies of predictive validity were found. Some of the tests were specific for indigenous populations, women, war veterans and schizophrenics. There are few instruments available for the assessment of resilience in adults and elderly people, especially for use in national studies.

Uniterms: Resilience, psychological; Studies; Psychological tests.

A resiliência pode ser caracterizada como a capacidade do ser humano em responder às demandas desfavoráveis da vida de maneira positiva. Isso resulta da associação entre os atributos do indivíduo e de seu ambiente familiar, social e cultural, e demonstra a capacidade de superação de condições adversas que poderiam ameaçar significativamente a sua saúde mental (Luthar, 2006; Masten, 2007; Rutter, 2006). Estima-se que, ao longo da vida, $51 \%$ das mulheres e $60 \%$ dos homens tenham vivenciado pelo menos um evento potencialmente traumático (Kessler, Sonnega, Bromet, Hughes \& Nelson, 1995).

O conceito de resiliência na Psicologia, historicamente, precedeu-se pelos termos invulnerabilidade e invencibilidade diante de fatores que pudessem causar stress ao indivíduo. Com o passar do tempo, esses termos tornaram-se inconsistentes, já que o stress é gradual e a resiliência é um fenômeno de interação do sujeito com o meio. Não há um consenso conceitual sobre o termo resiliência, devendo-se considerar a susceptibilidade individual (Oliveira, Reis, Zanelato \& Neme, 2008).

Segundo Noronha, Cardoso, Moraes e Centa (2009), a resiliência vem sendo estudada desde a década de 1970. As pesquisas, nessa época, buscavam identificar fatores de risco e proteção que se relacionavam aos aspectos de adaptação do indivíduo, e objetivavam subsidiar programas de intervenção e políticas públicas na saúde mental. A resiliência, nas ciências sociais e na saúde em geral, nas três últimas décadas, surge com grande importância no contexto das adversidades sociais, políticas e econômicas. Isso ocorre principalmente em regiões menos favorecidas e com problemas de difícil resolução (M. R. S. Silva, Lacharite, P. A. Silva, Lunardi \& Lunardi Filho, 2009). A utilização do conceito de resiliência em ambientes médico-psicossociais é mais recente e, atualmente, o termo busca incluir dimensões mais relacionadas às condições sociais (Noronha et al., 2009). Diante deste fato, evidencia-se a importância da resiliência e do desenvolvimento de instrumentos de avaliação desse construto.
Nessa perspectiva, a instabilidade do fenômeno resiliência constitui um desafio presente no processo de pesquisa, sobretudo no que se refere às formas de avaliar esse construto (Reppold, Mayer, Almeida \& Hutz, 2012). Dentre as principais formas utilizadas para avaliação da resiliência, podem-se citar: escores de testes (Connor \& Davidson, 2003) e outras medidas de desempenho (Brancalhone, Fogo \& Williams, 2004), perfis de personalidade ou temperamento (Pereira, 2001), análise de histórias de vida (Paludo \& Koller, 2005) e análise de discurso de pessoas que convivem com o sujeito (Riley et al., 2006). A maioria dessas medidas assume uma perspectiva individual para a avaliação da resiliência. Entretanto, Rutter (2006) ressalta que a avaliação da resiliência deve contemplar uma avaliação sistêmica, que considere as adversidades encontradas no contexto em que o sujeito está inserido.

Segundo estudo realizado por Souza e Cerveny (2006), a quantidade de artigos publicados sobre resiliência vem crescendo de maneira considerável, quase triplicando a cada cinco anos. A maior parte dos temas pesquisados refere-se aos efeitos da guerra e do holocausto (Job, 2001), ao impacto do abuso sexual na infância (Pinto Junior, 2001), ao uso de drogas por adolescentes (Schenker \& Minayo, 2005), aos efeitos de dependência química parental e do stress familiar (Figlie, Fontes, Moraes \& Paya, 2004), à relação entre violência e etnia (Vergara, 2008), ao desempenho acadêmico e ao divórcio (Marturano, 2008). Em alguns trabalhos, é evidente a prioridade de avaliações envolvendo traços de personalidade, características, resistência e enfrentamento (Peres, Mercante \& Nasello, 2005). Como delineamento de pesquisa, esses estudos variam entre estudos longitudinais (Werner, 1989), os quais definem a resiliência como um processo, priorizando nas avaliações o relativismo contextual, e estudos transversais (Gil \& Diniz, 2006), os quais buscam compreender a presença ou ausência de determinadas características psicossociais, eventos de vida ou traços de personalidade (Reppold et al., 2012; Souza \& Cerveny, 2006). 
Nesse sentido, é imprescindível ao desenvolvimento desses estudos a elaboração de novos instrumentos, que demonstrem evidências de validade e confiabilidade (Jordans, Komproe, Tol \& De Jong, 2009). Contudo, muitas das estratégias utilizadas com o objetivo de avaliar a resiliência não atingem esse propósito por não apresentar uma definição operacional adequada sobre o construto avaliado ou por utilizar métodos não validados como instrumentos de investigação. São poucos os testes validados para avaliação da resiliência disponíveis na literatura, embora o interesse pelo conceito de resiliência seja crescente e reflita o empenho da área na prevenção de problemas psicossociais e na promoção da saúde mental (Pesce et al., 2005). Portanto, é objetivo deste estudo realizar uma revisão sistemática acerca da validação dos instrumentos utilizados na literatura nacional e internacional para avaliação da resiliência de adultos e idosos em âmbito psicológico.

\section{Método}

Para investigação dos instrumentos disponíveis na literatura nacional e internacional que envolvessem a validação de instrumentos de avaliação de resiliência psicológica em adultos e idosos (indivíduos acima de 18 anos), foram pesquisadas as seguintes bases de dados eletrônicas (de janeiro de 1980 até março de 2010): MedLine (acessado pelo PubMed), PsycINFO, Scopus, SciElO e Interscience databases. Utilizou-se a combinação entre os termos de busca resilience, psychological, scales e validation studies e os respectivos termos em português e espanhol nas bases que utilizam essas línguas para indexação.

Foram incluídos todos os estudos de validação de instrumentos que envolviam a avaliação de resiliência psicológica em indivíduos acima de 18 anos (adultos e idosos). Os critérios de exclusão foram: não ter sido escrito em inglês, português ou espanhol, ou o estudo não se referir à validação de algum instrumento destinado à avaliação da resiliência. Os títulos e resumos de todos os artigos identificados pela estratégia de busca foram analisados pelos pesquisadores. Todos os resumos que preencheram suficientemente os critérios de inclusão foram selecionados para avaliação do texto integral. No estágio do texto integral, dois revisores independentemente avaliaram os artigos completos e realizaram suas seleções de acordo com os critérios de elegibilidade. Discordâncias entre os revisores foram resolvidas por consenso. Dois revisores independentes realizaram a coleta de dados no que diz respeito às características metodológicas, intervenções e desfechos dos estudos. O desfecho principal coletado foi a validação dos instrumentos de avaliação da resiliência entre adultos e idosos e a análise foi do tipo descritiva.

\section{Resultados}

Como resultado da busca inicial foram identificados 59 estudos, dentre os quais 16 atendiam aos critérios de inclusão e foram considerados como relevantes para a amostra deste trabalho, sendo analisados detalhadamente. Esses 16 estudos validavam 13 instrumentos diferentes com a função de avaliar a resiliência, ou continham a avaliação da resiliência em um de seus subtestes. São eles (e seus respectivos escores de validação): The Brief Resilience Scale (BRS) (alfa de Cronbach entre 0,80 e 0,91), validado por Smith et al. (2008); The Connor-Davidson Resilience Scale (CD-RISC) (alfa de Cronbach=0,89), validado por Connor e Davidson (2003); 10-item CD-RISC (alfa de Cronbach=0,85), validado por Campbell-Sills e Stein (2007); The Resilience Scale for Adults (RSA), validada por Friborg, Hjemdal, Rosenvinge e Martinussen (2003) (alfa de Cronbach entre 0,67 e 0,90) e por Hjemdal, Friborg, Stiles, Rosenvinge e Martinussen (2006) (alfa de Cronbach entre 0,81 e 0,88); Brief Resilient Coping Scale (alfa de Cronbach=0,69), validado por Sinclair eWallston (2004); Adolescent Resilience Scale, validado por Oshio, Kaneko, Nagamine e Nakaya (2003); Spanish Version of the Resilience Scale (alfa de Cronbach=0,93), validada por Heilemann, Lee e Kury (2003); Escala de Resiliência (alfa de Cronbach=0,91), validada por Pesce et al. (2005); Strong Souls (alfa de Cronbach $\geq 0,07$ ), validada por Thomas, Cairney, Gunthorpe, Paradies e Sayers (2010); Military Social Health Index (MSHI) (alfa de Cronbach=0,945), validado por Van Breda (2008); Deployment Risk and Resilience Inventory (DRRI), validado por D. W. King, L. A. King, Vogt, Knight e Samper (2006) (alfa de Cronbach entre 0,82 e 0,89) e por Vogt, Proctor, D. W. King, L. A. King e Vasterling (2008) (alfa de Cronbach $\geq 0,80$ ); Deployment Risk and Resilience 
Inventory in French-Canadian Veterans (alfa de Cronbach=0,85), validado por Fikretoglu, Brunet, Poundja, Guay \& Pedlar (2006); Patient-based health-related quality of life questionnaire in schizophrenia (S-QoL) (alfa de Cronbach=0,70), validado por Auquier et al. (2003); Chinese Positive Youth Development Scale (CPYDS) (alfa de Cronbach=0,91), validado por Shek et al. (2006).

O total de 7212 indivíduos foi incluído nos 16 estudos, e a idade dos indivíduos incluídos nas amostras dos trabalhos desta revisão variou de 12 a 97 anos. Os estudos foram divididos em duas categorias: uma relativa aos instrumentos validados com o objetivo principal de avaliar resiliência (Tabela 1) e outra referente aos instrumentos em que um de seus subtestes avaliava a resiliência (Tabela 2). Nas Tabelas 1 e 2 são apresentados os seguintes dados: autores do artigo, nome do instrumento, periódico em que foi publicado, língua original, ano de publicação, aspecto principal que avalia, idade da amostra, sexo da amostra e estudos psicométricos.

\section{Discussão e Conclusão}

Como observado neste estudo, existem poucas investigações priorizando métodos de adaptação transcultural e a investigação de índices psicométricos de

Tabela 1

Características dos estudos que validavam instrumentos cujo objetivo principal era a avaliação da resiliência. 1980-2010

\begin{tabular}{|c|c|c|c|c|c|c|c|}
\hline Artigo (autores) & Instrumento & Periódico & $\begin{array}{l}\text { Língua original; } \\
\text { ano de publicação }\end{array}$ & $\begin{array}{c}\text { Aspectos } \\
0 \text { que avalia }\end{array}$ & $\begin{array}{c}\text { Idade da } \\
\text { amostra e (n) }\end{array}$ & Sexo & Estudos psicométricos \\
\hline Smith et al. & $\begin{array}{c}\text { The Brief } \\
\text { Resilience Scale } \\
\text { (BRS) }\end{array}$ & $\begin{array}{l}\text { International } \\
\text { Journal of } \\
\text { Behavioral } \\
\text { Medicine }\end{array}$ & Inglês; 2008 & $\begin{array}{l}\text { Fatores de } \\
\text { resiliência }\end{array}$ & $\begin{array}{c}\text { Média de } 37,57 \\
\text { anos }(n=354)\end{array}$ & $\begin{array}{l}\text { Média de } \\
33,25(\mathrm{M}) \text { e } \\
66,75(\mathrm{~F})\end{array}$ & $\begin{array}{l}\text { Análise fatorial; } \\
\text { precisão; validade } \\
\text { convergente e } \\
\text { preditiva discriminante }\end{array}$ \\
\hline $\begin{array}{l}\text { Connor e } \\
\text { Davidson }\end{array}$ & $\begin{array}{l}\text { The Vonnor- } \\
\text { Davidson } \\
\text { Resilience Scale } \\
\text { (CD-RISC) }\end{array}$ & $\begin{array}{l}\text { Depression And } \\
\text { Anxiety }\end{array}$ & Inglês; 2003 & $\begin{array}{l}\text { Resiliência e } \\
\text { resposta ao } \\
\text { tratamento }\end{array}$ & $\begin{array}{l}\text { Média de 43,8 } \\
\text { anos (n=806) }\end{array}$ & $\begin{array}{c}35 \%(\mathrm{M}) \mathrm{e} \\
65 \%(\mathrm{~F})\end{array}$ & $\begin{array}{l}\text { Precisão; validade } \\
\text { convergente e } \\
\text { discriminante }\end{array}$ \\
\hline $\begin{array}{l}\text { Campbell-Sills } \\
\text { e Stein }\end{array}$ & 10-item CD-RISC & $\begin{array}{c}\text { Journal of } \\
\text { Traumatic Stress } \\
\text { International }\end{array}$ & Inglês; 2007 & $\begin{array}{l}\text { Funcionamento } \\
\text { positive } \\
\text { frente às } \\
\text { adversidades }\end{array}$ & $\begin{array}{l}\text { Média de 18,8 } \\
\text { anos (n=131) }\end{array}$ & $\begin{array}{l}25,6 \% \mathrm{M}) \mathrm{e} \\
74.4 \%(\mathrm{~F})\end{array}$ & $\begin{array}{l}\text { Análise fatorial; } \\
\text { validade de construto }\end{array}$ \\
\hline Friborg et al. & $\begin{array}{c}\text { The Resilience } \\
\text { Scale for Adults } \\
\text { (RSA) }\end{array}$ & $\begin{array}{l}\text { Journal of } \\
\text { Methods in } \\
\text { Psychiatric } \\
\text { Research }\end{array}$ & Inglês; 2003 & $\begin{array}{l}\text { Recursos de } \\
\text { proteção e } \\
\text { resiliência } \\
\text { em adultos }\end{array}$ & $\begin{array}{c}\text { Entre } 18 \text { e } 75 \\
\text { anos }(n=59)\end{array}$ & $\begin{array}{c}14(\mathrm{M}) \text { e } 45 \\
(\mathrm{~F})\end{array}$ & $\begin{array}{c}\text { Análise fatorial; } \\
\text { precisão; validade de } \\
\text { construto }\end{array}$ \\
\hline Hjemdal et al. & $\begin{array}{l}\text { The Resilience } \\
\text { Scale for Adults } \\
\text { (RSA) }\end{array}$ & $\begin{array}{c}\text { Clinical } \\
\text { Psychology and } \\
\text { Psychotherapy }\end{array}$ & Inglês;2006 & $\begin{array}{l}\text { Resiliência e } \\
\text { sintomas } \\
\text { psiquiátricos }\end{array}$ & $\begin{array}{l}\text { Média de 22,2 } \\
\text { anos ( } n=159)\end{array}$ & $\begin{array}{l}36(\mathrm{M}) \text { e } \\
123(\mathrm{M})\end{array}$ & $\begin{array}{c}\text { Precisão; Validade } \\
\text { preditiva }\end{array}$ \\
\hline $\begin{array}{l}\text { Sinclair e } \\
\text { Wallston }\end{array}$ & $\begin{array}{l}\text { Brief Resilient } \\
\text { Coping Scale } \\
\text { (BRCS) }\end{array}$ & Assessment & Inglês; 2004 & $\begin{array}{l}\text { Habilidades } \\
\text { de resiliência }\end{array}$ & $\begin{array}{l}\text { Média de } 46 \\
\text { anos }(n=230)\end{array}$ & $\begin{array}{l}\text { 27\% (M) e } \\
73 \%(F)\end{array}$ & $\begin{array}{l}\text { Precisão; Validade de } \\
\text { construto e preditiva }\end{array}$ \\
\hline Oshio et al. & $\begin{array}{c}\text { Adolescent } \\
\text { Resilience Scale }\end{array}$ & $\begin{array}{c}\text { Psychological } \\
\text { Reports }\end{array}$ & Inglês; 2002 & $\begin{array}{l}\text { Fatores de } \\
\text { resiliência } \\
\text { psicológica }\end{array}$ & $\begin{array}{l}\text { Entre } 19 \text { e } 23 \\
\text { anos }(n=207)\end{array}$ & $\begin{array}{c}104(\mathrm{M}) \mathrm{e} \\
103(\mathrm{~F})\end{array}$ & $\begin{array}{c}\text { Análise factorial; } \\
\text { precisão; validação de } \\
\text { construto }\end{array}$ \\
\hline $\begin{array}{l}\text { Heilemann } \\
\text { et al. }\end{array}$ & $\begin{array}{l}\text { Resilience Scale } \\
\text { (Spanish Version) }\end{array}$ & $\begin{array}{l}\text { Journal of } \\
\text { Nursing } \\
\text { Measurement }\end{array}$ & Inglês; 2003 & $\begin{array}{l}\text { Fatores de } \\
\text { resiliência }\end{array}$ & $\begin{array}{l}\text { Entre } 21 \text { e } 40 \\
\text { anos }(n=147)\end{array}$ & $\begin{array}{c}147(F) \text { e } \\
0(M)\end{array}$ & $\begin{array}{l}\text { Precisão; validade de } \\
\text { construto }\end{array}$ \\
\hline Pesce et al. & $\begin{array}{l}\text { Escala de } \\
\text { Resiliência }\end{array}$ & $\begin{array}{l}\text { Caderno de } \\
\text { Saúde Pública }\end{array}$ & Português; 2005 & $\begin{array}{l}\text { Fatores de } \\
\text { resiliência }\end{array}$ & $\begin{array}{l}\text { Entre } 12 \text { e } 19 \\
\text { anos }(n=997)\end{array}$ & $\begin{array}{l}52,2 \%(F) \text { e } \\
47,8 \%(M)\end{array}$ & $\begin{array}{c}\text { Análise fatorial; } \\
\text { precisão; validação de } \\
\text { conteúdo e construto }\end{array}$ \\
\hline
\end{tabular}

490

Nota: n: número amostral; nc: não consta; M: Masculino; F: Feminino. 
Tabela 2

Características dos estudos que validavam instrumentos em que um dos subtestes avaliava resiliência

\begin{tabular}{|c|c|c|c|c|c|c|c|}
\hline Artigo (autores) & Instrumento & Periódico & $\begin{array}{l}\text { Língua original; } \\
\text { ano de publicação }\end{array}$ & $\begin{array}{c}\text { Aspectos } \\
0 \text { que avalia }\end{array}$ & $\begin{array}{c}\text { Idade da } \\
\text { amostra e (n) }\end{array}$ & Sexo & Estudos psicométricos \\
\hline Thomas et al. & Strong Souls & $\begin{array}{l}\text { Australian and } \\
\text { New Zealand } \\
\text { Journal of } \\
\text { Psychiatry }\end{array}$ & Inglês; 2010 & $\begin{array}{l}\text { Bem-estar } \\
\text { social e } \\
\text { emocional }\end{array}$ & $\begin{array}{l}\text { Entre } 16 \text { e } 20,5 \\
\text { anos }(n=361)\end{array}$ & $\begin{array}{l}169,47 \%(M) \\
\text { e } 192,53(F)\end{array}$ & $\begin{array}{c}\text { Análise fatorial; } \\
\text { precisão; validação de } \\
\text { construto }\end{array}$ \\
\hline Van Breda & $\begin{array}{l}\text { Military Social } \\
\text { Health Index } \\
\text { (MSHI) }\end{array}$ & $\begin{array}{l}\text { Military } \\
\text { Medicine }\end{array}$ & Inglês; 2008 & $\begin{array}{l}\text { Resiliência } \\
\text { familiar }\end{array}$ & $n c(n=2000)$ & $\begin{array}{l}1464(\mathrm{M}) \\
532(\mathrm{~F})\end{array}$ & $\begin{array}{l}\text { Precisão; validade de } \\
\text { construto }\end{array}$ \\
\hline King et al. & $\begin{array}{l}\text { Deployment Risk } \\
\text { and Resilience } \\
\text { Inventory (DRRI) }\end{array}$ & $\begin{array}{c}\text { Military } \\
\text { Psychology }\end{array}$ & Inglês; 2006 & Resiliência & $\begin{array}{c}\text { Entre } 20 \text { e } \\
\text { maiores de } 60 \\
\text { anos }(n=357)\end{array}$ & $\begin{array}{l}75 \%(\mathrm{M}) \text { e } \\
25 \%(\mathrm{~F})\end{array}$ & $\begin{array}{c}\text { Precisão; Validade de } \\
\text { Construto }\end{array}$ \\
\hline Vogt et al. & $\begin{array}{l}\text { Deployment Risk } \\
\text { and Resilience } \\
\text { Inventory (DRRI) }\end{array}$ & Assessment & Inglês; 2008 & $\begin{array}{l}\text { Saúde e bem- } \\
\text { estar de } \\
\text { veteranos de } \\
\text { guerra }\end{array}$ & $\begin{array}{l}\text { Entre } 20 \text { e } 50 \\
\text { anos }(n=640)\end{array}$ & $\begin{array}{c}591(\mathrm{M}) \\
49(\mathrm{~F})\end{array}$ & $\begin{array}{l}\text { Precisão; validade de } \\
\text { critério }\end{array}$ \\
\hline Fikretoglu et al. & $\begin{array}{l}\text { Deployment Risk } \\
\text { and Resilience } \\
\text { Inventory (DRRI) }\end{array}$ & $\begin{array}{c}\text { The Canadian } \\
\text { Journal of } \\
\text { Psychiatry }\end{array}$ & Inglês; 2006 & $\begin{array}{l}\text { Risco de } \\
\text { sofrimento e } \\
\text { fatores de } \\
\text { resiliência }\end{array}$ & $\begin{array}{l}\text { Média de 49,94 } \\
\text { anos ( } \mathrm{n}=131)\end{array}$ & $\begin{array}{c}131(\mathrm{M}) \mathrm{e} \\
0(\mathrm{~F})\end{array}$ & $\begin{array}{c}\text { Precisão; validade de } \\
\text { critério }\end{array}$ \\
\hline Auquier et al. & $\begin{array}{c}\text { Patient-based } \\
\text { health- } \\
\text { relatedquality of } \\
\text { life questionnaire } \\
\text { in schizophrenia } \\
\text { (S-QoL) }\end{array}$ & $\begin{array}{l}\text { Schizophrenia } \\
\text { Research }\end{array}$ & Inglês; 2003 & $\begin{array}{l}\text { Bem-estar } \\
\text { psicológico e } \\
\text { resiliência }\end{array}$ & $\begin{array}{l}\text { Media de } 37,3 \\
\text { anos }(n=207)\end{array}$ & $\begin{array}{c}68,1 \%(\mathrm{M}) \text { e } \\
31,9(\mathrm{~F})\end{array}$ & $\begin{array}{l}\text { Precisão; validade de } \\
\text { contruto e conteúdo }\end{array}$ \\
\hline Shek et al. & $\begin{array}{c}\text { Chinese Positive } \\
\text { Youth } \\
\text { Development } \\
\text { Scale (CPYDS) }\end{array}$ & $\begin{array}{l}\text { International } \\
\text { Journal of } \\
\text { Adolescent } \\
\text { Medicine and } \\
\text { Health }\end{array}$ & Inglês; 2006 & $\begin{array}{l}\text { Desenvolvimento } \\
\text { positivo da } \\
\text { juventude }\end{array}$ & $\mathrm{nc}(\mathrm{n}=426)$ & $\begin{array}{c}242(\mathrm{M}) \mathrm{e} \\
184(\mathrm{~F})\end{array}$ & $\begin{array}{l}\text { Precisão; validade de } \\
\text { construto }\end{array}$ \\
\hline
\end{tabular}

Nota: n: número amostral; nc: não consta; M: Masculino; F: Feminino.

testes envolvendo a avaliação da resiliência (Pesce et al., 2005; Friborg, Barlaug, Martinussen, Rosenvinge \& Hjemdal, 2005). Oito instrumentos (validados em nove estudos) incluídos neste estudo foram desenvolvidos com o objetivo principal de avaliar a resiliência. Smith et al. (2008) validaram a BRS, uma escala de seis itens que tem por objetivo avaliar a capacidade do indivíduo de recuperar-se do estresse. Com relação à estrutura fatorial, os resultados demonstraram uma solução unifatorial com variância explicada de 55\%, cargas entre 0,68 e 0,91 e bom índice de consistência interna (alfa de Cronbach variando de 0,80 - 0,91 entre as diferentes amostras investigadas). No estudo original, a BRS obteve uma confiabilidade de teste-reteste de 0,69 e 0,61. Com relação à validade convergente, a BRS foi positivamente correlacionada com medidas de resiliência, otimismo, propósito na vida, apoio social e negativamente correlacionada com pessimismo, desajustamento comportamental, negação e autoculpa. Com relação à validação preditiva discriminante, numa amostra com pacientes cardíacos, observou-se que nas correlações de ordem-zero, a BRS foi correlacionada com sete desfechos, que são: percepção do estresse, ansiedade, depressão, afeto negativo, afeto positivo, fadiga e exercícios diários.

Connor e Davidson (2003) desenvolveram, na Inglaterra, a CD-RISC, que é composta por 25 itens. Esse estudo objetivou estabelecer valores de referência para - CD-RISC, avaliando a confiabilidade e validade da escala. A média dos escores foi calculada pelo agrupamento dos dados demográficos (sexo e raça - brancos 
e não brancos), não havendo diferenças entre as características avaliadas. Dentre as propriedades psicométricas, a consistência interna apresentou alfa de Cronbach de 0,89 e as correlações item-total variaram de 0,30 a 0,70. A confiabilidade no teste-reteste foi avaliada por meio de ensaio clínico com indivíduos com diagnóstico de Transtorno de Ansiedade Generalizada eTranstorno de Estresse Pós-traumático, obtendo coeficiente de correlação igual a 0,87. O estudo de validade convergente apontou que altos níveis de resiliência correlacionam-se com baixos níveis de vulnerabilidade ao estresse. A validade discriminante demonstrou que o CD-RISC não foi significativamente relacionado com a Escala de Experiência Sexual Arizona (McGahuey et al., 2000).

Campbell-Sills e Stein (2007), na Califórnia, trabalharam no aperfeiçoamento da CD-RISC, sugerindo uma versão de 10 itens da escala. Foi realizada inicialmente uma análise fatorial exploratória da versão original da CR-RISC, observando-se a inadequação de vários itens incluídos na versão original. A CD-RISC de 10 itens foi correlacionada a maus tratos na infância e sintomas psiquiátricos, a consistência interna foi boa, com alfa de Cronbach de 0,85. Com a redução para 10 itens, importantes características de resiliência ficaram de fora do instrumento, apesar de, ainda assim, a escala investigar de maneira satisfatória fatores de resiliência.

Friborg et al. (2003), na Noruega, validaram uma escala de 45 itens para mensurar a presença de recursos de proteção que promovem a resiliência em adultos: RSA. A versão preliminar abrange cinco dimensões: competência pessoal, competência social, coerência familiar, apoio social e estrutura pessoal. Essas dimensões foram indicadas por uma análise fatorial exploratória. A consistência interna, avaliada por meio do alfa de Cronbach, indicou valores entre 0,67 a 0,90. A correlação teste-reteste foi satisfatória (entre 0,69 e 0,84, $p<0,01)$. Com relação à validade de construto, esta escala foi positivamente relacionada com uma escala de senso de coerência e negativamente relacionada com um inventário de sintomas psiquiátricos. Hjemdal et al. (2006) utilizaram mais dois instrumentos para investigar a validade preditiva da RSA em relação à dor e ao stress, um questionário de avaliação de situações estressoras e um questionário de avaliação psiquiátrica, obtendo 492 correlações entre 0,74 e 0,84. Quanto ao índice de precisão desse estudo, obteve resultados similares ao estudo anterior, entre 0,81 e 0,88.

Sinclair e Wallston (2004) validaram, nos Estados Unidos, a Brief Resilient Coping Scale (BRCS), que é um instrumento de quatro itens, desenvolvido para avaliar tendências dos indivíduos para lidar com stress de uma forma adaptável. Utilizando uma amostra de 23 indivíduos com artrite reumatoide, a BRCS mostrou-se útil para identificar indivíduos com necessidades de melhorar as habilidades de enfrentamento do stress. Na análise da consistência interna, obteve-se alfa de Cronbach de 0,64 no início e 0,71 ao final dos três meses de acompanhamento dos pacientes.

Oshio et al. (2003) realizaram, no Japão, a validação de construto da Adolescent Resilience Scale. Esta escala foi desenvolvida por Oshio, Nakaya, Kaneko, e Nagamine (2002), em uma amostra de estudantes japoneses, e é composta por 21 itens e três fatores: busca de novidade, regulação emocional e orientação positiva para o futuro. Análises de correlação de Pearson demonstraram correlação positiva significativa entre todos os fatores desta escala. Os fatores propostos foram confirmados por análise fatorial. Quanto à consistência interna, os índices obtidos foram 0,85 para a pontuação total, 0,79 para procura de novidades, 0,77 para regulação emocional e 0,81 para orientação futura positiva.

Heilemann et al. (2003) validaram, na Califórnia, a tradução para o espanhol da RS, originalmente criada em inglês por Wagnild e Young (1993). Essa escala contém 25 itens e mensura o nível de adaptação psicossocial positiva em eventos importantes da vida. A confiabilidade do instrumento foi estimada com alfa de Cronbach de 0,93. Observou-se significativa correlação positiva entre resiliência e satisfação na vida e negativa entre resiliência e sintomas depressivos. A RS (Wagnild \& Young, 1993) é a única escala adaptada para o português, no Brasil, segundo as buscas realizadas. Foi adaptada por Pesce et al. (2005), em uma população de estudantes entre 12 e 19 anos. Na versão brasileira, os autores preocuparam-se em manter a equivalência semântica, operacional e de mensuração utilizadas na versão original. Os escores de alfa de Cronbach encontrados na versão brasileira se assemelham ao encontrado por Wagnild e Young (1993) (0,91), indicando boa consistência interna do instrumento. 
Foram encontrados, nos estudos incluídos neste trabalho, cinco outros instrumentos (validados em sete estudos) que consideravam a resiliência como um fator dos construtos investigados (ou seja, como um subteste). Um deles é o Strong Souls, instrumento desenvolvido por Thomas et al. (2010), na Austrália, para avaliar bem-estar social e emocional de jovens indígenas. Realizou-se validação de construto por meio de análise fatorial exploratória. Os resultados indicam que a solução fatorial de quatro fatores (bem-estar físico, emocional, social e espiritual) explica 34\% de variância. A análise da confiabilidade realizada sobre os 25 itens da escala e sobre cada fator apresentou índices de alfa de Cronbach $\geq 0,70$, sugerindo satisfatória consistência interna global da Strong Souls.

O estudo de Van Breda (2008) validou o Military Social Health Index (MSHI) no sul da África, um instrumento composto por quatro escalas, cada uma com 14 itens, que avaliam os seguintes fatores: suporte social, resolução de problemas, avaliação de stress e resiliência familiar/recursos de resistência. A resiliência familiar/ recursos de resistência é o foco principal da MSHI, referindo-se aos processos que as famílias usam para lidar de forma eficaz e resistente às crises. Os resultados iniciais da validação indicam que o MSHI demonstrou altos níveis de confiabilidade (média de 0,94). A média dos coeficientes de precisão foi de 0,73.

King et al. (2006), nos Estados Unidos, validaram a Deployment Risk and Resilience Inventory (DRRI). Essa escala tem 201 itens e baseia-se nos seguintes construtos: pré-implantação de fatores estressores, ambiente familiar na infância, fatores de difícil convivência, ambiente de trabalho, preocupações com relação à vida e rupturas familiares, assédio sexual, ameaça percebida, experiências de combate, consequências de batalha e consequências derivadas de exposições a armas nucleares, biológicas e químicas, fatores de suporte social e estressores pós-combate. A consistência interna para as medidas foi igual a 0,82 ou superior. Vogt et al. (2008), mais tarde, também nos Estados Unidos, realizaram estudo de validade da DRRI com uma amostra de 640 veteranos da guerra do Iraque. Com relação à consistência interna, sete dos dez fatores obtiveram coeficientes de alfa de Cronbach igual ou superior a 0,80. Os fatores com menos índice foram estressores prévios e exposição biológica, nuclear e química. Evidências de validade de critério foram obtidas a partir das correlações entre os escores da DRRI e os escores das medidas de saúde (sistema cardíaco, dermatológico, neurológico, pulmonar, gastrointestinal, músculo-esquelético e cansaço), de funcionamento social, problemas emocionais e percepção mental da saúde. A maioria dessas correlações alcançou significância esta-tística $(p<0,05)$. Sobre essa mesma escala, Fikretoglu et al. (2006), no Canadá, validaram a versão francesa/canadense da escala Deployment risk and resilience factors (DRRI) e examinaram as relações entre riscos, fatores de resiliência e funcionamento pós-combate em uma amostra de 131 indivíduos. Os coeficientes alfa da DRRI apresentaram um escore de 0,85, em média, e variaram de 0,67 a 0,94. As estimativas de confiabilidade teste-reteste pela correlação de Pearson variaram de 0,69 a 0,93 para cada um dos 14 itens.

Auquier et al. (2003) desenvolveram e validaram o Patient-based Health-related Quality of Life Questionnaire in Schizophrenia (S-QoL), na França, como um instrumento autoaplicado que avalia a qualidade de saúde e qualidade de vida em pessoas com diagnóstico de esquizofrenia. Essa escala contém 41 itens envolvendo bem-estar psicológico (dez itens), autoestima (seis itens), família (cinco itens), relacionamentos com amigos (cinco itens), resiliência (cinco itens), bem-estar físico (quatro itens), autonomia (quatro itens) e vida sentimental (dois itens). Os índices de consistência interna dos fatores variaram de 0,63 a 0,90. Os autores concluíram que o impacto da esquizofrenia na vida dos indivíduos poderia ser adequadamente mensurado com o S-QoL. Shek et al. (2006), em Hong Kong, desenvolveram a Chinese Positive Youth Development Scale (CPYDS). Essa escala envolve 15 aspectos do desenvolvimento positivo de jovens, incluindo vínculos (seis itens), resiliência (seis itens), competência social (sete itens), competência emocional (seis itens), competência cognitiva (seis itens), competência moral (seis itens), competência comportamental (seis itens), autodeterminação (cinco itens), autoeficácia (sete itens), espiritualidade (sete itens), visão de futuro (sete itens), autoidentificação positiva (sete itens), envolvimento social (cinco itens), normas sociais (cinco itens) e reconhecimento de comportamentos positivos (quatro itens). Com relação ao total da escala (90 itens), a correla- 
ção interitem foi de 0,40, e o coeficiente alfa da escala total foi de 0,91. Enquanto a CPYDS foi positivamente correlacionada com desenvolvimento, bem-estar e com medidas de satisfação com a vida, foi negativamente correlacionada com abuso de substâncias, delinquência e intenção de engajar-se em comportamentos antissociais. Os dados indicam a confiabilidade e validação da CPYDS com jovens chineses.

Observa-se que, dos estudos que utilizavam instrumentos de avaliação específica de resiliência, $77,7 \%$ incluíram indivíduos do sexo feminino como maioria em suas amostras. Já os estudos que utilizavam instrumentos em que a avaliação da resiliência era um subteste, apresentaram, em 87,5\% dos estudos, uma quantidade maior de indivíduos do sexo masculino (geralmente militares ou ex-combatentes de guerra). A literatura sugere que não existem diferenças significativas entre os níveis de resiliência de homens e mulheres: o que se observa é um perfil diferente de resiliência em ambos os sexos (Guajardo \& Paucar, 2008). Sugere-se que mais estudos sejam realizados validando esses instrumentos que incluem a resiliência como um subteste em amostras femininas. Em relação ao número de itens, os instrumentos que avaliavam resiliência em um subteste apresentaram um número menor de itens de resiliência (média de 13,1 itens) em comparação àqueles que utilizavam instrumentos específicos para avaliar esse construto (média de 22,8 itens). Quanto ao fator idade, os estudos distribuíram-se de maneira adequada entre as faixas etárias que compreendem a idade adulta e idosa. Os estudos de Campbell-Sills e Stein (2007) e de King et al. (2006) foram os únicos que relacionaram a resiliência dos adultos e idosos aos maus tratos e ambiente familiar na infância.

Apenas dois dos instrumentos são voltados para o desenvolvimento de adultos jovens. Um deles (Oshio et al., 2003) relacionado a estudantes e sua regulação emocional e orientação positiva para o futuro; e o outro (Thomas et al., 2010) voltado especificamente para a população de adultos jovens indígenas.

Todos os estudos apresentaram seus dados psicométricos de maneira satisfatória, revelando qualidade ao avaliar os fatores aos quais se propunham. Os principais indicadores psicométricos dos instrumentos foram estudos de precisão (em $87,7 \%$ dos estudos) e validade de construto (em 68,7\% dos estudos). Três dos instrumentos (Heilemann et al., 2003; Fikretoglu et al., 2006; Pesce et al., 2005) foram também adaptados para línguas diferentes da versão original (espanhol, francês e português, respectivamente), apresentando, segundo dados psicométricos, tradução adequada e equivalência de termos, evitando a interferência de erro no uso do teste. Apenas um foi validado para uso no Brasil (Pesce et al., 2005).

Observou-se que poucos estudos preditivos empíricos foram apresentados investigando a relação entre resiliência, questões de saúde geral e desenvolvimento psicossocial. Esses dados podem refletir a dificuldade dos teóricos em operacionalizar a definição do construto resiliência. Sugere-se, assim, que mais estudos preditivos empíricos sejam realizados com populações de adultos e idosos.

Os resultados também apontam para a necessidade de desenvolvimento e validação de novos instrumentos que possam avaliar construtos relacionados à perspectiva do ajustamento emocional sobre situação de risco, bem como a necessidade de tradução e validação para o português da maioria dos instrumentos apresentados nesta revisão.

Sugere-se a criação de instrumentos nacionais para avaliação da resiliência, dada a carência de instrumentais observada. Como limitação, ressalta-se que este estudo incluiu apenas artigos em que os autores utilizaram os termos (descritores) de busca mencionados, e que a não utilização de descritores adequados pelos autores dos artigos originais ou a ausência dos descritores no título ou no resumo dos artigos pode ter excluído algum trabalhos pela dificuldade de identificação nas bases utilizadas para a pesquisa. Sugere-se que mais revisões sistemáticas da literatura sejam realizadas envolvendo também o processo de desenvolvimento da resiliência nos indivíduos e o efeito de diferentes intervenções terapêuticas sobre a mesma.

Por fim verificou-se que a resiliência é avaliada na literatura por meio de poucos instrumentos validados. Os estudos baseiam-se em perspectivas teóricas diversas e muitos associam a resiliência à qualidade de vida, satisfação, saúde e bem-estar, risco, resistência e desenvolvimento positivo. Os instrumentos incluídos foram desenvolvidos para a população adulta e idosa, embora se deva considerar que muitos estudos re- 
ferem-se a populações específicas, tais como as indígenas, mulheres, japoneses e veteranos de guerra. Assim, os estudos de validação da resiliência mostram-se escassos na literatura, e fica evidente a necessidade de mais pesquisas envolvendo esta temática, bem como a necessidade de instrumentos adaptados à realidade brasileira.

\section{Referências}

Auquier, P., Simeoni, M. C., Sapin, C., Reine, G., Aghababian, V., \& Cramer (2003). Development and validation of a patient-based health-related quality of life questionnaire in schizophrenia:The S-QoL. Schizophrenia Research, 1(63), 137-149.

Brancalhone, P. G., Fogo, J. C., \& Williams, L. C. A. (2004). Crianças expostas à violência conjugal: avaliação do desempenho acadêmico. Psicologia: Teoria e Pesquisa, 20(2), 113-117.

Campbell-Sills, L., \& Stein, M. B. (2007). Psychometric analysis and refinement of the Connor-Davidson Resilience Scale (CD-RISC): Validation of a 10-item measure of resilience. Journal of Traumatic Stress, 20(6), 1019-1028.

Connor, K. M., \& Davidson, J. R. T. (2003). Development of a new resilience scale: The Connor-Davidson Resilience Scale (CDRISC). Depression and Anxiety, 18(2), 76-82.

Figlie, N., Fontes, A., Moraes, E., \& Paya, R. (2004). Filhos de dependentes químicos com fatores de risco bio-psicossociais: necessitam de um olhar especial? Revista de Psiquiatria Clínica, 31(2), 53-62.

Fikretoglu, D., Brunet, A., Poundja, J., Guay, S., \& Pedlar, D. (2006). Validation of the deployment risk and resilience inventory in French-Canadian veterans: Findings on the relation between deployment experiences and postdeployment health. Canadian Journal of Psychiatry, 51(12), 755-763.

Friborg, O., Barlaug, D., Martinussen, M., Rosenvinge, J. H., \& Hjemdal, O. (2005). Resilience in relation to personality and intelligence. International Journal of Methods in Psychiatric Research, 14(1), 29-42.

Friborg, O., Hjemdal, O., Rosenvinge, J. H., \& Martinussen, M. (2003). A new rating scale for adult resilience: What are the central protective resources behind healthy adjustment? International Journal of Methods in Psychiatric Research, 12(2), 65-76.

Gil, G., \& Diniz, J. A. (2006). Educadores de infância promotores de saúde e resiliência: um estudo exploratório com crianças em situação de risco. Análise Psicológica, 24(2), 217-234.

Guajardo, E. S., \& Paucar, M. V. (2008). Medición de lãs características resilientes, un estudio comparativo en personas entre 15 y 65 años. Liberabit, 14(14), 31-40.

Heilemann, M. V., Lee, K., \& Kury, F. S. (2003). Psychometric properties of the Spanish version of the Resilience Scale. Journal of Nursing Measurement, 11(1), 61-72.
Hjemdal, O., Friborg, O., Stiles, T. C., Rosenvinge, J. H., \& Martinussen, M. (2006). Resilience predicting psychiatric symptoms: A prospective study of protective factors and their role in adjustment to stressful life events. Clinical Psychology \& Psychotherapy, 13(3), 194-201.

Job, J. R. P. P. (2001). A escritura da resiliência: testemunhas do holocausto e a memória da vida (Tese de doutorado não- publicada). Programa de Pós-Graduação em Psicologia, Pontifícia Universidade Católica de São Paulo.

Jordans, M. J., Komproe, I. H., Tol, W. A., \& De Jong, J. T. (2009). Screening for psychosocial distress amongst war-affected children: Cross-cultural construct validity of the CPDS. The Journal of Child Psychology and Psychiatry, 50(4), 514-523.

Kessler, R. C., Sonnega, A., Bromet, E., Hughes, M., \& Nelson, C. B. (1995). Posttraumatic stress disorder in the National Comorbidity Survey. Archives of General Psychiatry, 52(12), 1048-1460.

King, L. A., King, D. W., Vogt, D. S., Knight, J., \& Samper, R. E. (2006). Deployment risk and resilience inventory: A collection of Measures for studying deployment-related experiences of military personnel and veterans. Military Psychology, 18(2), 89-120.

Luthar, S. S. (2006). Resilience in development: A synthesis of research across five decades. In D. Cicchetti \& D. J. Cohen (Orgs.), Developmental psychopathology: Risk, disorder and adaptation (pp.739-795). New York: Wiley.

Marturano, E. M. (2008). Tensões cotidianas na transição da primeira série: um enfoque de desenvolvimento. Psicologia em Estudo, 13(1), 79-87.

Masten, A. S. (2007). Resilience in developing systems: Progress and promise as the fourth wave rises. Development and Psychopathology, 19(3), 921-930.

McGahuey, C. A., Gelenberg, A. J., Laukes, C. A., McKnight, K. M., Manber, R., \& Moreno, F. A. (2000). The Arizona Sexual Experiences Scale (ASEX): Reliability and validity. Journal of Sex and Marital Therapy, 26(1), 25-40.

Noronha, M. G. R. C. S., Cardoso, P. S., Moraes, T. N.P., \& Centa, M. L. (2009). Resilience: A new perspective in health promotion? Ciência \& Saúde Coletiva, 14(2), 497-506.

Oliveira, M. A., Reis, V. L., Zanelato, L. S., \& Neme, C. M. B. (2008). Resiliência: análise das publicações no período de 2000 a 2006. Psicologia: Ciência e Profissão, 28(4), 754-767.

Oshio, A., Kaneko, H., Nagamine, S., \& Nakaya, M. (2003). Construct validity of the adolescent resilience scale. Psychological Reports, 93(3f), 1217-1222.

Oshio, A., Nakaya, M., Kaneko, H., \& Nagamine, S. (2002). Development and validation of an adolescent resilience scale. Japanese Journal of Counseling Science, 35, 57-65.

Paludo, S. S., \& Koller, S. H. (2005). Resiliência na rua: um estudo de caso. Psicologia: Teoria e Pesquisa, 21(2), 187-195.

Pereira, A. M. S. (2001). Resiliência, personalidade, stress e estratégias de coping. In J. Tavares (Org.), Resiliência e educação (pp.77-94). São Paulo: Cortez.

Peres, J. F. P., Mercante, J. P. P., \& Nasello, A. G. (2005). Promovendo resiliência em vítimas de trauma psicológico. Revista de Psiquiatria do Rio Grande do Sul, 27(2), 131-138. 
Pesce, R. P., Assis, S. G., Avanci, J. Q., Santos, N. C., Malaquias, J. V., \& Carvalhaes, R. (2005). Adaptação transcultural, confiabilidade e validade da escala de resiliência. Caderno de Saúde Pública, 21(2), 436-448.

Pinto Junior, A. A. (2001). O trabalho com crianças vítimas de violência sexual doméstica: promovendo a resiliência. Temas Sobre Desenvolvimento, 10(55), 40-46.

Reppold, C. T., Mayer, J. C., Almeida, L. S., \& Hutz, C. S. (2012). Avaliação da resiliência: controvérsia em torno do uso de escalas. Psicologia: Reflexão e Crítica, 25(2), 248-255.

Riley, A. W., Coghill, D., Forrest, C. B., Lorenzo, M. J., Ralston, S. J., \& Spiel, G. (2006). Validity of the health-related quality of life assessment in the ADORE study: Parent report form of the CHIP-Child Edition. European Child \& Adolescent Psychiatry, 15(1 Suppl.), 63-71.

Rutter, M. (2006). Implications of resilience concepts for scientific understanding. Annals of the New York Academy of Sciences, 1094(1), 1-12.

Schenker, M., \& Minayo, M. C. S. (2005). Fatores de risco e de proteção para o uso de drogas na adolescência. Ciência \& Saúde Coletiva, 10(3), 707-717.

Shek, D. T. L., Siu, A. M. H., Lee, T. Y., Cheng, H., Tsang, S., Lui, J. et al. (2006). Development and validation of a positive youth development scale in Hong Kong. International Journal of Adolescent Medicine and Health, 18(3), 547-558.

Silva, M. R. S., Lacharite, C., Silva, P. A., Lunardi, V. L., \& Lunard Filho, W. D. (2009). Processos que sustentam a resiliência familiar: um estudo de caso. Texto \& Contexto: Enfermagem, 18(1), 92-99.

Sinclair, V. G., \& Wallston, K. A. (2004). The development and psychometric evaluation of the Brief Resilient Coping Scale. Assessment, 11(1), 94-101.
Smith, B. W., Dalen, J., Wiggins, K., Tooley, E., Christopher, P., \& Bernard, J. (2008). The brief resilience scale: Assessing the ability to bounce back. International Journal of Behavioral Medicine, 15(3), 194-200.

Souza, M. T. S., \& Cerveny, C. M. O. (2006). Resiliência psicológica: revisão da literatura e análise da produção científica. Revista Interamericana de Psicología, 40(1), 115-122.

Thomas, A., Cairney, S., Gunthorpe, W., Paradies, Y., \& Sayers, S. (2010). Strong Souls: Development and validation of a culturally appropriate tool for assessment of social and emotional well-being in Indigenous youth. Australian and New Zealand Journal of Psychiatry, 44(1), 40-8.

Van Breda, A. D. (2008). The military social health index: A partial multicultural validation. Military Medicine, 173(5), 480-487.

Vergara, S. C. (2008). A resiliência de profissionais angolanos. Revista de Administração Pública, 42(4), 701-718.

Vogt, D. S., Proctor, S. P., King, D. W., King, L. A., \& Vasterling, J. J. (2008). Validation of scales from the deployment risk and resilience Inventory in a sample of operation Iraqi freedom veterans. Assessment, 15(4), 391-403.

Wagnild, G. M., \& Young, H. M. (1993). Development and psychometric evaluation of the resilience scale. Journal of Nursing Measurement, 1(2), 165-168.

Werner, E. E. (1989). High-risk children in young adulthood: A longitudinal study from birth to 32 years. American Journal of Orthopsychiatry, 59(1), 72-81.

Recebido em: 6/6/2011

Versão final em: 12/12/2011

Aprovado em: 12/3/2012 